\title{
Prevalência de sobrepeso e obesidade em escolares do município de Feliz, Rio Grande do Sul, Brasil
}

Prevalence of overweight and obesity in schoolchildren in the municipality of Feliz, Rio Grande do Sul state, Brazil

Prevalencia de sobrepeso y obesidad en niños escolares del municipio de Feliz, Rio Grande do Sul, Brasil

Paulo Ricardo Panazzolo. Hospital do Círculo Operário Caxiense. Caxias do Sul, RS, Brasil. paullorp2@ig.com.br (Autor correspondente) Helius Carlos Finimundi. Universidade de Caxias do Sul (UCS). Caxias do Sul, RS, Brasil. helius@bitcom.com.br

Maria Otilia Spier Stoffel. Hospital Municipal Schlatter. Feliz, RS, Brasil. ticanutri@pop.com.br

Ronie André Simon. Hospital Municipal Schlatter. Feliz, RS, Brasil. ronieandresimon@gmail.com

Márcia Cristina de Lima. Hospital Municipal Schlatter. Feliz, RS, Brasil. marciacristinadelima3@gmail.com

Cristine Bonne Costanzi. Universidade de Caxias do Sul (UCS). Caxias do Sul, RS, Brasil. cristine.costanzi@gmail.com

\section{Resumo}

Objetivo: Determinar a prevalência de sobrepeso e obesidade entre escolares do ensino fundamental no município de Feliz-RS, no ano de 2010.

Métodos: Se realizou um censo escolar que incluiu 633 alunos com idades entre 6 e 10 anos, tendo sido feita avaliação antropométrica que incluía peso e altura. Para a classificação de sobrepeso e obesidade, foram utilizados pontos de corte propostos pelo Centres for Disease Control and Prevention (CDC) 2000, sendo considerado sobrepeso um índice de massa corpórea (IMC) entre o percentil 85 e menor que 95 e obesidade um IMC percentil $\geq 95$. Resultados: A prevalência de obesidade no grupo foi de $15,9 \%$ e 0 sobrepeso acometeu $15,4 \%$ das crianças. Somando-se as duas condições, $31,4 \%$ dos escolares estavam acima do peso esperado para a idade e estatura. A prevalência de obesidade foi maior nas faixas etárias de 6 e 7 anos, quando comparada às das crianças de 9 e 10 anos, respectivamente, 23,3\% e 8,23\% ( $p<0,001)$. Meninos e meninas não apresentaram diferença significativamente estatística na prevalência de sobrepeso (15,4\% para 15,2\%) e obesidade (16,1\% para 15,2\%), respectivamente. Em relação à localização geográfica, meninos locados em escolas na zona rural do município apresentaram maior prevalência de obesidade quando comparados aos meninos das escolas localizadas na sede do município, respectivamente $22,7 \%$ para $13,6 \%$ ( $p=0,036)$. Conclusão: A obesidade foi significativamente mais prevalente nas crianças de 6 e 7 anos de idade e também nos meninos matriculados nas escolas da zona rural do município.

\section{Abstract}

Objective: To determine the prevalence of overweight and obesity amongst elementary schoolchildren in the municipality of Feliz, Rio Grande do Sul state, in 2010. Methods: An anthropometric assessment, which included weight and height, was carried out through a school census including 633 6 to 10-years-old students. The cut-off points proposed by the Centres for Disease Control and Prevention (CDC) 2000 were used for classification of overweight and obesity, defining overweight as a body mass index (BMI) between the 85 and less than 95 percentile, and obesity as a BMI above the 95 percentile. Results: Obesity prevalence was found in $15.9 \%$ and overweight in $15.4 \%$ of the children in the group; therefore, $31.4 \%$ of the schoolchildren surveyed were above the expected weight and height for their age when both conditions were combined. Obesity prevalence was higher in children aged 6 to 7 years old when compared with children aged 9 to 10 years old, $23.3 \%$ and $8.23 \%(p<0.001)$, respectively. Boys and girls did not present statistically significant difference in prevalence of overweight (15.4\% and $15.2 \%$ ) and obesity (16.1\% and 15.2\%), respectively. Regarding geographical location, the boys from schools in the countryside of the municipality presented a higher prevalence of obesity when compared with the boys from schools in the urban area, $22.7 \%$ and $13.6 \%(p=0.036)$, respectively. Conclusion: Obesity was significantly more prevalent in children aged 6 to 7 years-old and in boys enrolled in schools in the rural area of the municipality.

Como citar: Panazzolo PR, Finimundi HC, Stoffel MOS, Simon RA, Lima MC, Costanzi CB.

Palavras-chave:

Sobrepeso

Obesidade

Saúde da Criança

Prevalência

Keywords:

Overweight

Obesity

Child Health

Prevalence 


\section{Resumen}

Objetivo: determinar la prevalencia de sobrepeso y obesidad entre escolares de educación primaria del municipio de Feliz-RS, en el año 2010. Métodos: se realizó un censo escolar, que incluyó a 633 alumnos con edades entre los 6 y los 10 años, se hizo la evaluación antropométrica que incluía peso y altura. Para la clasificación de sobrepeso y obesidad se utilizaron los puntos de corte propuestos por el Centres for Disease Control and Prevention (CDC) 2000, que considera el índice de masa corporal (IMC) comprendido entre los percentiles 85 y menor que 95 como sobrepeso, y el percentil mayor que 95 como índice de obesidad. Resultados: la prevalencia de obesidad fue de 15,9\% y la de sobrepeso fue de $15,4 \%$. Sumando las dos condiciones, el 31,4\% de los escolares estaban por encima del peso apropiado para su edad y estatura. La prevalencia de la obesidad fue mayor en el rango etario de los 06 a 07 años que en el rango de 09 a 10 años, con una puntuación de 23,3\% y 8,23\% respectivamente $(p<0,001)$. Los niños, de ambos sexos, no mostraron ninguna diferencia estadísticamente significativa ni en la prevalencia de sobrepeso (de 15,4\% y $15,2 \%$ ) ni en la de obesidad (de 16,1\% y 15,2\%) respectivamente. Respecto a la ubicación geográfica, los niños de las escuelas situadas en las zonas rurales del municipio presentaron una mayor prevalencia de obesidad que los niños de escuelas localizadas en la sede del municipio, de $22,7 \%$ y $13,6 \%$ respectivamente $(\mathrm{p}=0,036)$. Conclusión: La obesidad fue significativamente más frecuente en los niños entre 6 y 7 años de edad y también en los niños de sexo masculino matriculados en las escuelas de las áreas rurales del municipio.
Palabras clave:

Sobrepeso

Obesidad

Salud del Niño

Prevalencia

\section{Introdução}

Nos últimos 30 anos, a incidência de obesidade em crianças cresceu de menos de $5 \%$ para aproximadamente $20 \%$ nos Estados Unidos. ${ }^{1}$ No Brasil, estima-se que $33 \%$ das crianças entre 5 a 9 anos de idade estejam acima do peso, sendo que destas 14,3\% são consideradas obesas, conforme Pesquisa de Orçamentos Familiares em 2008 e 2009. ${ }^{2}$ Crianças com obesidade e sobrepeso apresentam maior incidência de fatores de risco para o desenvolvimento de doença arterial coronariana, devido a alteraçóes metabólicas que se manifestam, mais frequentemente detectados na idade adulta, como alterações dos lípides séricos, metabolismo da glicose, hipertensão arterial e anormalidades da coagulação. ${ }^{3}$

O risco de a obesidade na primeira década de vida persistir na idade adulta está relacionado ao tempo, à duraçáo da condição e à sua gravidade. Com o avanço da idade e o aumento do grau da obesidade as taxas de remissão diminuem e o risco de persistência aumenta. ${ }^{4,5}$ Para as crianças obesas após os 6 anos de idade, a probabilidade de desenvolverem obesidade na idade adulta é muito maior quando os pais também são obesos: se ambos os pais forem obesos, a criança apresenta $80 \%$ de probabilidade de ser obesa; quando um dos pais é obeso, a chance cai para 50\%; e quando nenhum dos pais é obeso a chance da criança desenvolver obesidade é de $9 \% .^{6-8}$

As graves consequências da obesidade, as dificuldades para o seu controle, o aumento expressivo de sua prevalência nas diversas faixas etárias e o alto custo gerado para a sociedade, fazem dela um relevante problema de saúde pública que precisa ser abordado desde as idades mais precoces.

$\mathrm{Na}$ criança, a obesidade pode estar associada a um aumento de problemas respiratórios (asma, apneia do sono), problemas musculoesqueléticos, doença hepática, problemas comportamentais, isolamento social, sinais de depressão, baixa autoestima, bullying e piora do desempenho escolar. ${ }^{9,10}$

O presente estudo teve por objetivo avaliar a prevalência de sobrepeso e obesidade em escolares de 6 a 10 anos de idade, matriculados no ensino fundamental do município de Feliz, localizado no Vale do Caí, próximo à região metropolitana de Porto Alegre-RS, no ano de 2010.

\section{Métodos}

Foi realizado um censo escolar dos alunos matriculados nas instituiçóes de ensino fundamental no Município de Feliz-RS, no ano de 2010, com idade de 6 anos em março de 2010 e menos de 11 anos no mesmo mês. O município de Feliz está localizado no Vale do Caí, próximo à regiáo metropolitana de Porto Alegre, tem aproximadamente 12.500 habitantes, com uma área de $96,2 \mathrm{~km}^{2}$ e uma população de descendência predominantemente Germânica (75\%). A economia baseia-se na agricultura familiar, comércio e indústria e apresenta um índice de desenvolvimento humano (IDH) de 0,839.

A população do estudo formou-se a partir dos dados fornecidos pela Secretaria de Educação do Município e foram complementados pelos estabelecimentos de ensino, totalizando 654 crianças matriculadas no ensino fundamental do município, locadas em 10 escolas, sendo 4 escolas na zona urbana e 6 escolas na zona rural. Mesmo em maior número, as escolas do interior do município respondiam por apenas $31 \%$ das crianças matriculadas nas escolas de ensino fundamental. 


\section{Critérios para inclusão no estudo:}

- Estar matriculado em instituição de ensino fundamental no Município de Feliz no ano de 2010.

- Idade entre 72 e 131 meses, tomando-se como base o mês de março de 2010.

- Apresentar o Termo de Consentimento Livre e Esclarecido (TCLE) assinado pelos pais ou responsáveis.

\section{Critérios para exclusão do estudo:}

- Crianças com necessidades especiais.

- Crianças cujos pais não assinaram o TCLE.

- Crianças fora da faixa etária estipulada no estudo.

A avaliação antropométrica foi feita exclusivamente pelos pesquisadores, sempre no interior das escolas e em sala reservada. Foram registrados o peso em quilogramas e a altura em centímetros, utilizando os mesmos equipamentos: estadiômetro modelo II com base suporte e balança eletrônica da marca Welmy, com precisão de $100 \mathrm{~g}$.

A partir do cálculo do IMC, dividindo o peso em quilogramas pela altura em metros elevada ao quadrado $\left(\mathrm{m}^{2}\right)$, as crianças foram classificadas conforme a tabela proposta pelo Centres for Disease Control and Prevention (CDC) 2000 e determinado percentil, com os seguintes pontos de corte: ${ }^{11}$

- Sobrepeso: IMC percentil $\geq 85$ e $<95$

- Obesidade: percentil $\geq 95$

- Crianças com percentil $<5$ foram classificadas como de baixo peso, e crianças com percentil entre 5 e 85 foram classificadas como de peso normal.

\section{Aspectos éticos}

Este estudo foi aprovado pelo Comitê de Ética em Pesquisa em Seres Humanos do Hospital Nossa Senhora do Pompeia de Caxias do Sul-RS, parecer consubstanciado n ${ }^{\circ}$ 92/2010 de acordo com a resolução n 196/96 do Conselho Nacional de Saúde. Todos os participantes foram informados dos objetivos do projeto, concordaram e assinaram o Termo de Consentimento Livre e Esclarecido (TCLE).

\section{Análise estatística}

Para a análise estatística, foi utilizada inicialmente a estatística descritiva, agrupando os resultados em valores da média e desvio padráo. Para as variáveis contínuas com distribuiçáo normal, foi usado o Teste t de Student náo pareado para comparação da média entre os grupos. A análise dos dados foi realizada pelo pacote estatístico SPSS, versão 16.0 e foi adotado nível de significância de $\mathrm{p}<0,05$.

\section{Resultados}

Das 654 crianças matriculadas nas escolas de ensino fundamental do município de Feliz no ano de 2010 e que preencheram os critérios de inclusão no estudo, 21 não foram avaliadas (18 não consentiram e 3 faltaram nos dias da avaliação), totalizando entáo 633 crianças avaliadas, o que corresponde a 96,7\% do total dos alunos matriculados em toda a rede de ensino fundamental do município. O número de meninos foi ligeiramente maior do que o das meninas, totalizando $53,2 \%$ dos alunos, porém não houve diferença significativamente estatística entre os dois grupos. A maioria das crianças estava matriculada em escolas da zona urbana do município, 432 alunos ou 68,2\% do total avaliado, sendo que 201 alunos frequentavam escolas do interior do município (Tabela 1). 
Tabela 1. Características das crianças matriculadas em escolas do município de Feliz-RS, 2010.

\begin{tabular}{lccc}
\hline Características & Meninas n (\%) & Meninos $\mathrm{n}(\%)$ & Valor $\mathrm{p}$ \\
\hline Sexo & $296(46,8 \%)$ & $337(53,2 \%)$ & NS \\
Idade* (meses) $_{\text {Escola Zona Urbana }}$ & $103 \pm 16$ & $101 \pm 17$ & $\mathrm{NS}$ \\
Escola Zona Rural & $205(69 \%)$ & $227(67 \%)$ & $\mathrm{NS}$ \\
Escola Pública & $91(31 \%)$ & $110(33 \%)$ & $\mathrm{NS}$ \\
Escola Privada & $256(86 \%)$ & $300(89 \%)$ & $\mathrm{NS}$ \\
\end{tabular}

*Média \pm Desvio Padrão n (\%)= Frequência e Percentual NS= Não Significância Estatística.

A prevalência de excesso de peso encontrada nos alunos do ensino fundamental do município de Feliz no ano de 2010 foi de $31,4 \%$ quando consideradas as duas condiçóes juntas e somadas (sobrepeso e obesidade), tendo sido incluídos todos os alunos com IMC no percentil acima de $85 \%$. Ao separar os dois grupos, 15,4\% dos alunos estavam na faixa de sobrepeso e $15,9 \%$ classificados como obesos, apresentando estes últimos um IMC no percentil maior que $95 \%$. A maioria das crianças $(65,2 \%)$ encontrava-se com peso normal e apenas 3,3\% apresentavam IMC no percentil menor que 5\%, classificadas como de baixo peso (Tabela 2).

Meninas e meninos não apresentaram diferença significativamente estatística para as duas condiçóes avaliadas no estudo, sobrepeso e obesidade, tendo sido respectivamente $15,5 \%$ para $15,4 \%$ e $15,2 \%$ para $16,6 \%$ (Tabela 2).

Tabela 2. Prevalência de sobrepeso e obesidade em escolares de acordo com a localização e tipo de estabelecimento de ensino, Feliz-RS, 2010.

\begin{tabular}{|c|c|c|c|c|c|c|}
\hline \multirow[t]{2}{*}{ Escola } & \multicolumn{3}{|c|}{ Sobrepeso } & \multicolumn{3}{|c|}{ Obesidade } \\
\hline & $\begin{array}{c}\text { Meninas } \\
\text { n (\%) }\end{array}$ & $\begin{array}{c}\text { Meninos } \\
\text { n (\%) }\end{array}$ & Valor $p$ & $\begin{array}{c}\text { Meninas } \\
\text { n (\%) }\end{array}$ & $\begin{array}{c}\text { Meninos } \\
\mathrm{n}(\%)\end{array}$ & Valor $\mathrm{p}$ \\
\hline Cidade $(n=432)$ & $32(15,6)$ & $35(15,4)$ & NS & $31(15,1)$ & $31(13,6)$ & $N S$ \\
\hline Interior $(n=201)$ & $14(15,4)$ & $17(15,4)$ & NS & $14(15,3)$ & $25(22,7)$ & $N S$ \\
\hline Público $(n=556)$ & $40(15,6)$ & $45(15)$ & $N S$ & $40(15,6)$ & $52(17,3)$ & $N S$ \\
\hline Privado $(\mathrm{n}=77)$ & $6(15)$ & $7(18,9)$ & NS & $5(12,5)$ & $4(10,8)$ & $N S$ \\
\hline Total $(n=633)$ & $46(15,5)$ & $52(15,4)$ & NS & $45(15,2)$ & $56(16,6)$ & $N S$ \\
\hline
\end{tabular}

$\mathrm{n}(\%)$ = Frequência e Percentual NS= Não significância estatística.

A prevalência de obesidade nas escolas públicas foi 29,3\% maior que nas escolas privadas, porém não houve significância estatística na amostra avaliada, principalmente pelo pequeno número de crianças avaliadas em escola privada.

Dos meninos matriculados em escolas localizadas na zona rural do município, 22,7\% foram classificados como obesos, índices estes 39,8\% maiores do que os meninos que estudavam na sede do município, em que 13,6\% foram classificados como obesos $(\mathrm{p}=0,036)$.

As crianças de 6 e 7 anos apresentaram maior prevalência de obesidade quando comparadas às crianças de 9 e 10 anos, sendo respectivamente $23,3 \%$ para $8,2 \%$ com $\mathrm{p}<0,001$; o mesmo não aconteceu com as crianças classificadas na faixa de sobrepeso, em que não houve uma diferença significativamente estatística entre o grupo de crianças mais jovens em relação às crianças de 9 e 10 anos (Gráfico 1).

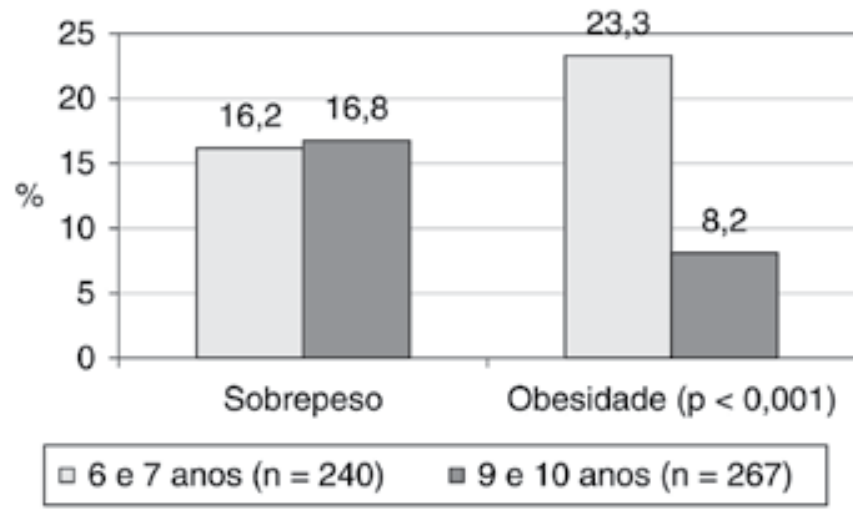

Gráfico 1. Prevalência de sobrepeso e obesidade nas diferentes faixas etárias dos escolares, Feliz-RS, 2010. 


\section{Discussão}

Dados brasileiros com relação à obesidade infantil são ainda limitados e a ausência de unanimidade na definição de obesidade, assim como a metodologia utilizada na avaliação desta faixa etária, acarreta dificuldades na comparação das prevalências relatadas nos diversos estudos. Visando avaliaçôes futuras e a adoção de medidas de controle e monitoramento nutricional da criança, aqui focados no sobrepeso e obesidade na infância como fatores de risco importantes para a morbimortalidade por doenças cardiovasculares na idade adulta, optou-se por um estudo na forma de censo escolar no Município de Feliz, no ano de 2010, o que possibilitou a avaliação de $96,7 \%$ de todos os escolares com critérios de inclusão para o estudo, possibilitando uma ampla análise dos resultados, seja na totalidade ou por faixa etária, ou mesmo das diferentes instituiçóes de ensino (públicas e privadas), locadas tanto na sede municipal quanto no meio rural.

Para esta avaliação, optou-se pela utilização do IMC por idade, método recomendado pela International Obesity Task Force (IOTF) por sua alta validade na estimativa de obesidade infantil em nível populacional. Para tanto, foram utilizados os valores de corte propostos pelo CDC 2000. ${ }^{11}$ É sabido que o método escolhido tem limitaçôes, sendo o IMC largamente utilizado para avaliação de sobrepeso e obesidade em adultos por possuir uma boa relação com medidas mais apuradas da gordura corporal. Deve ser utilizado, porém, julgamento clínico para a aplicação desses critérios, não apenas na criança. $\mathrm{O}$ termo obesidade se refere mais ao excesso de adiposidade do que propriamente ao excesso de peso, e o IMC é usado como uma ferramenta de substituição para medida da adiposidade corporal, e, mesmo que corrigido por tabelas, poderia ter maior sensibilidade e especificidade para detecção do excesso de adiposidade quando associado a outros métodos náo invasivos, como a medida da circunferência abdominal e/ou prega cutânea. ${ }^{12}$

Os resultados do estudo, no seu objetivo principal, sem considerar análise de subgrupos, apresenta-se em concordância com o relatório do IBGE, do período de 2008-2009, em que a prevalência excesso de peso nas crianças de 5 a 9 anos foi de $33,5 \%$, sendo que destes, $14,3 \%$ foram considerados obesos. No presente estudo, $31,4 \%$ das crianças de 6 a 10 anos encontravam-se acima do peso esperado, e destas, $15,9 \%$ foram consideradas obesas.

A maior prevalência de obesidade entre crianças de 6 e 7 anos segue um padrão semelhante ao de outros estudos, como os realizados na cidade de Londrina, em $2005,{ }^{13}$ e na cidade de Sáo Paulo, no ano de $2007 .{ }^{14}$ Estes também relataram taxas de prevalência distintas entre as idades, com maior predomínio de sobrepeso e obesidade nas crianças de 6 e 7 anos quando comparadas às crianças de 9 e 10 anos. Não existem, porém, até o momento, dados epidemiológicos que confirmem maiores índices de obesidade nas crianças dessa faixa etária.

A diversidade de populaçóes incluídas nos estudos ao longo do tempo, o processo de transição nutricional de muitas famílias, principalmente as de menor renda, e os métodos utilizados para avaliar sobrepeso e obesidade em crianças sáo fatores que dificultam a determinação dos índices de sobrepeso e obesidade nas diferentes faixas etárias. Novos estudos feitos nessa mesma populaçáo poderâo inferir maior valor aos achados aqui relatados.

As várias fases de crescimento da criança acarretam maior ou menor acúmulo de gordura em determinado tempo. Crianças crescem em tempos diferentes, algumas têm desenvolvimento puberal mais precoce em relação a outras, e essa diferença torna-se mais aparente na puberdade. ${ }^{15} \mathrm{O}$ início da puberdade geralmente ocorre na idade de 9 anos em meninas americanas e 11 anos em meninos americanos. ${ }^{16} \mathrm{O}$ presente estudo incluiu um número significativo de crianças com idade maior que 10 anos e menor que 11 anos, o que pode ter sido um dos fatores a influenciar esses menores índices de obesidade nessa faixa etária, pois algumas crianças já estariam numa fase de crescimento acelerado, próximo aos primeiros estágios da puberdade.

Não há no momento um consenso com relação a índices maiores de excesso de peso em meninos ou meninas. Alguns estudos apontam para maior prevalência de excesso de peso no gênero masculino. ${ }^{14,17} \mathrm{O}$ presente estudo não mostrou diferenças significativas entre os sexos. Alguns autores questionam se mudanças de hábitos alimentares e tipos de brincadeiras semelhantes, adotadas por meninos e meninas, poderiam ter influência sobre estes resultados, pois em tempos passados meninos adotavam brincadeiras com maior gasto energético do que as meninas. ${ }^{14}$

O processo de transição nutricional caracterizado pela urbanização, migração campo-cidade, industrialização, acesso mais facilitado aos alimentos de alto valor calórico, por vezes pobres em fibras e de baixo custo, além do fácil acesso ao transporte e, no caso das crianças, mudanças de hábitos quanto à prática de atividade física - que hoje por questōes de segurança ficam mais restritas ao lar - parece ter alterado o perfil nutricional e epidemiológico desta populaçáo nas últimas 2 ou 3 décadas, no Brasil. ${ }^{18,19}$ 
Em relação ao meio em que a criança estuda, o tipo de escola (pública ou privada), e a localização desses estabelecimentos, não houve diferença significativamente estatística na prevalência de sobrepeso e obesidade nas crianças locadas em instituiçóes públicas ou privadas, porém esta análise ficou prejudicada pelo pequeno número de crianças locadas em escolas privadas, apenas $31 \%$ do total. Estudos realizados em Santos ${ }^{16}$ Salvador $^{20}$ e Pelotas ${ }^{21}$ avaliaram escolares de faixas etárias semelhantes, locados em instituições públicas e privadas, mostraram maior prevalência de sobrepeso e obesidade entre os alunos das escolas privadas. Essa maior prevalência da obesidade nas instituições privadas pode ser explicada pela associação entre excesso de gordura corporal e níveis socioeconômicos mais elevados, o que ainda é esperado em países em desenvolvimento. Entretanto, a transição nutricional que vem ocorrendo nestes países demonstra que a prevalência de obesidade está crescendo em todos os estratos socioeconômicos, e mais predominantemente nas classes sociais de baixa renda e baixa escolaridade. No Brasil, atualmente observa-se um aumento proporcionalmente mais elevado da prevalência de obesidade nos indivíduos adultos de famílias de menor poder aquisitivo, o que revela uma proporção mais alta de obesos na classe média do que nas classes de maior poder aquisitivo.

Seguindo um padrão compatível com país em desenvolvimento, o estudo mostrou maior prevalência de obesidade entre as crianças locadas em escolas na zona rural do município, principalmente em meninos que apresentaram prevalência de 22,7\% de obesidade contra 13,6\% para os meninos que residiam na sede do município. Uma hipótese para esta diferença seriam as condiçôes econômicas das crianças que vivem no meio rural, geralmente oriundas de famílias com menos acesso à educação, que trabalham no setor primário e têm renda familiar menor.

De maneira interessante, em países subdesenvolvidos, pessoas com maior acesso à educação e condições econômicas mais favoráveis estáo mais propensas à obesidade do que pessoas de nível socioeconômico e cultural mais baixo. Situação inversa é percebida em países desenvolvidos, nos quais pessoas com maior poder econômico e maior acesso à educação tem taxas menores de obesidade do que as pessoas mais pobres. Em uma situação intermediária, situam-se países ditos 'em desenvolvimento', caso de China e Brasil. ${ }^{22,23}$

Estudo com crianças de 6 a 10 anos realizado na cidade de São Paulo, ${ }^{14}$ somente em escolas privadas de diferentes classes sociais, encontrou maior prevalência de obesidade nos estabelecimentos que abrigavam crianças de nível socioeconômico mais baixo. Grandes estudos norte-americanos vinculam maiores taxas de sobrepeso e obesidade a grupos socioeconômicos e culturais menos favorecidos ou etnias específicas. ${ }^{24,25}$

Os achados deste estudo reforçam a ideia de que o excesso de peso na população é um problema de saúde pública que se inicia precocemente na infância e tende a se perpetuar na idade adulta, influenciando de maneira direta e imediata a qualidade de vida destas populaçôes, aumentando a morbimortalidade pelas doenças crônicas não transmissíveis (DCNT), principalmente doenças cardiovasculares e neoplasias. A detecçáo precoce do excesso de peso ou obesidade na criança no ambiente escolar favorece o monitoramento e a adoção de medidas preventivas e educativas em tempo hábil, dando um prognóstico mais favorável de forma mais sustentada e duradoura a longo prazo.

\section{Conclusão}

O excesso de peso esteve presente em 31,4\% dos alunos matriculados nas escolas de ensino fundamental do município de Feliz no ano de 2010, sendo que $15,9 \%$ dos alunos foram classificados como obesos. A obesidade foi significativamente mais prevalente nas crianças de 6 e 7 anos de idade e também nos meninos matriculados nas escolas da zona rural do município. No presente estudo, a escola mostrou ser local adequado para a detecção precoce do excesso de peso na criança com um custo praticável, mesmo em larga escala, podendo assim auxiliar os órgáos de saúde pública na adoçáo de medidas preventivas, tratamento e monitoramento nutricional das crianças, com reflexos diretos sobre a prevalência das doenças crônicas não transmissíveis e obesidade na idade adulta. 


\section{Referências}

1. Ogden CL, Carroll MD, Curtin LR, Lamb MM, Flegal KM. Prevalence of high body mass index in US children and adolescents, $2007-2008$. JAMA. 2010;303(3):242-249. PMid:20071470. http://dx.doi.org/10.1001/jama.2009.2012

2. Ministério do Planejamento, Orçamento e Gestão (BR), Instituto Brasileiro de Geografia e Estatística (IBGE). Pesquisa de orçamentos familiares 2008-2009: antropometria e estado nutricional de crianças, adolescentes e adultos no Brasil. Rio de Janeiro: IBGE; 2010. Disponível em: http://www.ibge.gov.br/home/estatistica/populacao/condicaodevida/pof/2008_2009_encaa/pof_20082009_encaa.pdf.

3. Kristine N. Obesity and coronary artery disease. In: Foody MJ. Preventive cardiology: strategies for the prevention and treatment of coronary artery disease. New Jersey: Humana Press; 2001. chapt. 09, p. 159-173. Disponível em: http://elibrary.rajavithi.go.th/homelibrary/E-book9/Foody_Preventive\%20Cardiology_0896038114.pdf.

4. Escrivão Schmidt MAM. Prevenção da obesidade. In: Palma D, Escrivão Schmidt MAM, Oliveira FLC. Guias de medicina ambulatorial e hospitalar da UNIFESP-EPM: nutrição clínica na infância e na adolescência. São Paulo: Manole; 2009. cap. 23, p. 355.

5. Gee M, Mahan KL, Escott-Stump S. Controle de peso em crianças. In: Mahan KL, Escott-Stump S. Krause alimentos nutrição e dietoterapia. $12^{a}$ ed. Rio de Janeiro: Elsevier; 2010. cap. 21, p. 556-557.

6. Ribeiro PRQ, Borges GC. Prevalência de sobrepeso e obesidade infantil em uma escola pública de Patos de Minas. Rev. Min. Enferm. 2010;(2)109-118. Disponível em: http://revistasaude.unipam.edu.br/edicao-n.-2/-out.-2010.

7. Kaufman A. Obesidade infanto-juvenil. Pediatr. Mod. 1999;35(4):218-22. Disponível em: http://www.moreirajr.com.br/revistas.asp?fase=r003\&id_materia=936.

8. Carvalhal MIM, Silva AJ, Miranda Neto JT. Clarificação do conceito de obesidade e sobrecarga e definição das consequências. In: Silva AJ, Miranda JT Neto, Raposo JJBV, Carvalhal, MIM. Obesidade Infantil. Montes Claros: CGB Artes Gráficas; 2007. cap. 2, p. 37-78.

9. Gahagan S. Overweight and obesity. In: Kliegman RM, Stanton BF, Behrman RE, Geme JWS, Schor NF. Nelson textbook of pediatrics. 19th ed. Philadelphia: Elsevier/Saunders; 2011. chapt. 44, p. 179-188. http://dx.doi.org/10.1016/B978-1-4377-0755-7.00044-0

10. Lumeng CJ, Forrest P, Appugliese DP, Kaciroti N. Weight status as a predictor of being bullied in third through sixth grades. Pediatrics. 2010;125(6):1301-1307. PMid:20439599. http://dx.doi.org/10.1542/peds.2009-0774

11. Kuczmarski RJ, Ogden CL, Guo SS, Grummer-Strawn LM, Flegal KM, Mei Z, et al. 2000 CDC growth charts for the United States: methods and development. Vital Health Stat. 2002;11(246):1-190. Disponível em: http://www.cdc.gov/growthcharts.

12. American Academy of Pediatrics; Committee on Nutrition. Prevention of pediatric overweight and obesity. Pediatrics. 2003;112(2):424-430. http://dx.doi.org/10.1542/peds.112.2.424

13. Ronque VER, Cyrino ES, Dorea VR, Serassuelo H Jr, Galdi EHG, Arruda M. Prevalence of overweight and obesity in schoolchildren of high socioeconomic level in Londrina, Paraná, Brazil. Rev Nutr. 2005;18(6):709-717.

14. Padua ECR, Martins EE, Ferreira MFA, Santilli PN, Costa RF, Fisberg M. Diagnóstico do estado nutricional de crianças de 6-10 anos em escolas da rede privada de ensino da cidade de São Paulo. Int Rev Nutr. 2008;1(1):14-20.

15. Kimmel SR, Ratliff-Schaub, K. Pubertal growth and development. In: Rakel RE, Rakel DP. Textbook of family medicine. 8th ed. Philadelphia: Elsevier/Saunders; 2011. p. 424-426. chapt. 23.

16. Tanner JM, Whitehouse $\mathrm{RH}$. Clinical longitudinal standards for height, weight, height velocity, weight velocity, and stages of puberty. Arch Dis Child. 1976;51:170-179. http://dx.doi.org/10.1136/adc.51.3.170

17. Costa RF, Cintra IP, Fisberg M. Prevalência de sobrepeso e obesidade em escolares da cidade de Santos. Arquivos Bras Endocrinol Metab. 2006;50(1):60-67. http://dx.doi.org/10.1590/S0004-27302006000100009

18. Souza EB. Transição nutricional no Brasil: análise dos principais fatores. Cad. UniFOA. 2010;(13)49-53. Disponível em: http://www.unifoa.edu.br/cadernos/edicao/13/49.pdf.

19. Popkin BM. The nutrition transition and its health implications in lower-income countries. Public Health Nutr. 1998;1(1):5-21. PMid:10555527. http://dx.doi.org/10.1079/PHN19980004

20. Leão LCS, Araújo LM, Moraes LP, Assis AM. Prevalência de obesidade em escolares de Salvador Bahia. Arq Bras Endocrinol Metab. 2003;47(2):151-157. http://dx.doi.org/10.1590/S0004-27302003000200007

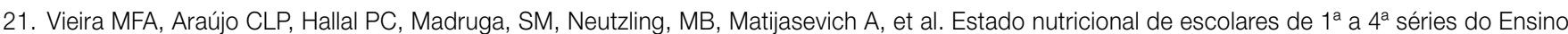
Fundamental das escolas urbanas da cidade de Pelotas, Rio Grande do Sul, Brasil. Cad. Saúde Pública. 2008;24(7):1667-1674. http://dx.doi.org/10.1590/S0102-311X2008000700021

22. Monteiro CA, Conde WL, Popkin BM. Income-specific trends in obesity in Brazil: 1975-2003. Am J Public Health. 2007;97(10):1808-1812. PMid:17761560 PMCid:PMC1994168. http://dx.doi.org/10.2105/AJPH.2006.099630

23. Popkin BM. Does global obesity represent a global public health challenge? Am J Clin Nutr. 2011;93(2):232-3. PMid:21159790 PMCid:PMC3021421. http://dx.doi.org/10.3945/ajcn.110.008458

24. Ogden CL, Flegal KM, Carroll MD, Johnson CL. Prevalence and trends in overweight among US children and adolescents, $1999-2000$. JAMA. 2002;288(14):1728-32. PMid:12365956. http://dx.doi.org/10.1001/jama.288.14.1728

25. Centers for Disease Control and Prevention. CDC grand rouns: childhood obesity in the United States. JAMA. 2011;305(10):988-991. Disponível em: http://jama.jamanetwork.com/article.aspx?articleid=646070. PMid:21248681. 